\title{
LAS POLÍTICAS PÚBLICAS DE FORMACIÓN DOCTORAL Y SU IMPACTO EN EL SISTEMA NACIONAL DE CIENCIA, TECNOLOGÍA E INNOVACIÓN. UNA LECTURA COMPARATIVA DE ARGENTINA Y BRASIL*
}

\section{THE PUBLIC POLITICS OF DOCTORAL FORMATION AND HIS IMPACT IN THE NATIONAL SYSTEM OF SCIENCE, TECHNOLOGY AND INNOVATION. A READING COMPARATIVE OF ARGENTINA AND BRAZIL}

\section{LUCAS ARIEL OVIEDO}

Facultad de Ciencias Económicas

Universidad Nacional del Nordeste

ARGENTINA

"¿De qué utilidad podrá servir para el común de nuestros labradores el que un compatriota se forme en el silencio de su gabinete, que atesore sólo para sí, que no difunda y propague aquellos conocimientos que adquirió, y que unos libros tan útiles se hallen sólo circunscriptos a la pequeña esfera de un estante?". 


\title{
RESUMEN
}

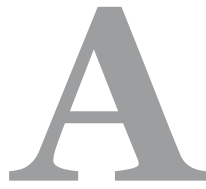

partir de información disponible en la Red de Indicadores de Ciencia y Tecnología -Iberoamericana e Interamericana- (RICYT), en este trabajo nos aproximamos a comprender el impacto que, en términos de resultados de productos científicos y tecnológicos (publicaciones en Science Citation Index -SCI- y solicitudes de patentes), han tenido las políticas públicas de formación doctoral implementadas en la última década en Argentina y Brasil. La lectura diacrónica y cuantitativa de la relación formación doctoral y resultados del sistema de ciencia y tecnología (SCT) se basa en el tradicional modelo "insumo-producto" utilizado en los estudios macro-económicos del nivel de producción de las actividades científicas y tecnológicas. El análisis se complementa con una mirada estructural del SCT latinoamericano, el cual presenta un conjunto de rasgos que condicionan las posibilidades de cambios sistémicos de este tipo de políticas públicas. En los últimos años tanto Argentina como Brasil han incrementado su población de doctores, lo que ha incidido de manera diferenciada en un aumento en las publicaciones científicas de sus comunidades académicas. Esto no ha ocurrido en términos de creatividad tecnológica, donde los resultados son escasos. Precisamente, un impacto de estas características parecería obedecer a las bajas inversiones del sector privado en ciencia y tecnología, a la escasa demanda de conocimiento científico-tecnológico de este sector y al bajo grado de articulación entre las instituciones públicas de $\mathrm{CyT}$ y el sector empresario, entre otros rasgos estructurales del sistema.

Palabras clave: políticas públicas, formación doctoral, sistema nacional de ciencia y tecnología.

\begin{abstract}
On the basis of information available on the Network of Science and Technology Indicators - Iberoamericana and inter-American- (RICYT), in this work we approach to understand the impact that, in terms of results of scientific and technological products (publications in Science Citation Index -SCI- and patent applications), have had the doctoral training public policies implemented in the last decade in Argentina and Brazil. The diachronic and quantitative reading of the doctoral training relationship and results of the system of science and technology (SCT) is based on the traditional model "input-output" used in the macroeconomic studies of the production level of scientific and technological activities. The analysis is complemented with a structural look of the SCT Latin American, which presents a set of
\end{abstract}

"Este artículo fue producido en el marco del Proyecto de Investigación PI M002-13 "Contextos de trabajo: entramados, poder y violencia. Estudios sobre organismos del estado, programas sociales, sector rural y cambio tecnológico" (periodo 2014-2017) del Grupo de Investigación "Trabajo, Sociedad y Subjetividad" de la FCE UNNE que integra el autor y que dirige la Dra. Ana Rosa Pratesi. El PI está acreditado por Resolución N839/13 del Consejo Superior de la UNNE. 
traits that affect the possibilities of systemic changes of this type of public policies. In recent years both Argentina and Brazil have increased their population of Ph.D, which has impacted differently in an increase in the scientific publications of their academic communities. This has not happened in terms of technological creativity, where the results are scarce. Precisely, an impact of these characteristics would seem to be due to the low private sector investments in science and technology at the low demand of scientific and technological knowledge of this sector and the low degree of articulation among the public institutions of S\&T and the business sector, among other structural features of the system.

Keywords: public policies, doctoral formation, national system of science and technology.

\section{INTRODUCCIÓN}

En este trabajo se realiza un análisis comparativo de los resultados en términos de productos científicos y tecnológicos que han tenido las políticas públicas orientadas a la formación doctoral en Argentina y Brasil. Para ello se describe el desempeño que, en la última década, han presentado dos indicadores de productos de ciencia y tecnología (CyT) -publicaciones en Science Citation Index (SCI) y solicitudes de patentes- elaborados por la Red de Indicadores de Ciencia y Tecnología -Iberoamericana e Interamericana- (RICYT). La lectura diacrónica y cuantitativa de la relación formación doctoral y resultados del sistema de ciencia y tecnología (SCT) se basa en el tradicional modelo "insumo-producto" utilizado en los estudios macro-económicos del nivel de producción de las actividades científicas y tecnológicas. Ahora bien, las explicaciones en las diferencias o similitudes en el rendimiento de ambos países se explican por las características estructurales que ha tenido desde sus orígenes el SCT en América Latina y el Caribe (ALC). En esta línea, la hipótesis que guía el presente trabajo refiere al tipo de condicionamiento que tiene, y ha tenido, el modelo de producción de CyT en ALC, el cual tradicionalmente se ha caracterizado por un bajo desempeño en términos de inversión, la baja participación del sector privado en el financiamiento de la I+D, la escasa articulación entre los organismos de CyT y el sector productivo y rasgos particulares que presentan las comunidades científicas locales y sus vínculos con el poder político. Así, el real impacto de las políticas públicas de formación de nivel doctoral implementadas en Brasil y Argentina se vieron limitadas por estos factores estructurales.

Es de destacar que estos países representan dos de las principales economías de ALC. Ambos países iniciaron en la década de 1950 su trayectoria en el campo de la promoción de la CyT, constituyéndose como los más relevantes de la región en la producción de conocimiento científico y tecnológico. Por ello, el análisis de las políticas públicas orientadas a promover la formación de profesionales altamente calificados de estos países reviste particular interés para la comparación. 


\section{POLÍticas PÚBLICAS ORIENTADAS A LA FORMACIÓN DOCTORAL. ¿POR QUÉ Y PARA QUÉ FORMAR DOCTORES?}

Los programas de formación de posgrado de nivel doctoral representan una herramienta ampliamente utilizada en ALC como parte de las políticas de CyT y de educación superior. Con diferencias institucionales, en todos los países se evidencia la necesidad de formar profesionales altamente calificados con el propósito principal de favorecer el desarrollo económico, productivo y socio-cultural. A partir de la década de los noventa, en la región se produjo un incremento considerable en el número de estudiantes de posgrado. En los inicios de esa década, García Guadilla (1996) construye una tipología de países de acuerdo al número de la matrícula de los estudiantes de posgrado, identificando cuatro grandes grupos: 1) Brasil y México, que cuentan con una población de posgrado entre 30 mil y 50 mil estudiantes; 2) Argentina, Chile, Colombia, Perú y Venezuela con una población de estudiantes entre 4.5 y 7 mil; 3) Bolivia, Costa Rica y Cuba con una población entre mil y 3 mil, y; 4) por último, el resto de los países con una población de posgrado de menos de 500 (García Guadilla, ibíd., p. 53).

Una década más tarde, Rama Vitale (2007) afirma que la población de estudiantes de posgrado en ALC alcanza a más de 400 mil alumnos, triplicando la población de los noventa. De este análisis se observa que Brasil y México continúan liderando el ranking en cuanto a población de estudiantes de posgrado (representan un poco más del 50\% de la población), mientras que el resto de las naciones concentra la otra mitad. Más allá del peso de Brasil y México, los datos actuales indican que el resto de los países elevaron considerablemente su población de estudiantes, lo que demuestra que la formación de posgraduados ha sido objeto de política pública en toda la región.

Para Mollis (2010), esta transformación no sólo refleja un incremento cuantitativo en la población de estudiantes de posgrado, sino también un cambio cualitativo en los mismos de la mano de los procesos de privatización de la educación superior. Esta autora sostiene que, en estas dos últimas décadas, en la región se produjo una tensión entre la formación en investigación y el entrenamiento profesional en áreas especializadas a raíz de la explosión institucional y matricular de estos últimos con relación a los primeros. Este proceso se ve acompañado por un cambio significativo en la relación entre el peso relativo del sector público y del sector privado en toda la educación superior y también en los posgrados en la región (RamaVitale, op. cit.).

Asimismo, es necesario comprender que la consolidación de la comunidad científica de un país está asociada a la formación de recursos humanos y a la consolidación de instituciones del conocimiento y de grupos de investigadores comprometidos con uno o varios temas de investigación en los cuales han demostrado capacidad de producción de resultados a través de publicaciones, obtención de patentes o comercialización de los productos de investigación (Jaramillo Salazar, 2009). Así, el discurso de la política sobre la formación de posgrado de 
nivel doctoral se ha consolidado de la mano de los procesos de maduración de los sistemas científicos y tecnológicos en la región, los que se produjeron en el marco de los debates teóricos respecto de la transición social hacia el desarrollo de estas naciones.

Como es sabido, la CyT se han convertido en objeto de política pública en el contexto de la Segunda Guerra Mundial, que es cuando estas dos actividades comienzan a ser concebidas como las grandes fuerzas impulsoras del desarrollo económico y social de las naciones (Albornoz, 2011). Específicamente, se reconoce a Vannevar Bush ${ }^{1}$ como el gran ideólogo de este proceso, al reconocer el peso de las ciencias básicas por sobre las restantes ciencias, y como el camino inicial para el desarrollo tecnológico. Se inicia la primacía de un modelo lineal ${ }^{2} \mathrm{de}$ difusión social del conocimiento que comienza con la ciencia básica y finaliza con la tecnología. Posteriormente, a partir de los años ochenta, la globalización de la economía transforma el discurso en torno a la CyT. De la mano de conceptos como "sociedad del conocimiento" y "economía del conocimiento", los organismos internacionales comienzan a difundir la idea de que la creación, acumulación y circulación del conocimiento favorece el desarrollo de la competitividad de los países. Al binomio I+D se le anexa una "i” minúscula en representación del concepto nodal de la época: la innovación.

De ahora en más, las políticas públicas de CyT buscan promover el desarrollo de los Sistemas Nacionales de Innovación (SIN), entendiendo al fenómeno innovativo como el resultado de un proceso interactivo y de aprendizaje entre actores, visión superadora del modelo lineal de producción del conocimiento. En este marco, la formación de profesionales altamente calificados representa un insumo fundamental para el desarrollo de los SIN, ya que se considera que la escasez de profesionales en ciencia e ingeniería puede afectar significativamente la expansión de industrias competitivas (Global Science Forum, 2008).

Luchilo (2010) señala que, en sus inicios, la carrera de posgrado apuntaba a la formación de científicos capaces de mover las fronteras de la ciencia a través de sus investigaciones en el campo de las ciencias básicas, cuyo mecanismo de comunicación de resultados ha sido tradicionalmente la publicación de papers. Posteriormente, el énfasis se trasladó a la búsqueda del conocimiento aplicado y al desencadenamiento de procesos innovadores en las empresas, siendo las patentes su mejor forma de expresión. Sin embargo, a pesar del incremento en el número de posgrados en ALC, Mollis y Jaim Etcheverry (2003) sugieren que no se ha producido el impacto esperado en cuanto a la producción de conocimiento innovador y la capacitación de quienes se dedican a la CyT. Esta falta de correlación tiene diversas aristas y una de ellas se explica por las características estructurales que ha adquirido el complejo de CyT en ALC.

\footnotetext{
${ }^{1}$ En el año 1945 Vannevar Bush, en su carácter de Director de la Oficina de Investigación y Desarrollo Científico de los Estados Unidos, elaboró un informe al entonces presidente de ese país titulado "Ciencia, la frontera sin fin”. En ese informe Bush afirmaba que más y mejor ciencia significará el progreso de toda la sociedad.

${ }^{2}$ Este modelo lineal, también representado como modelo ofertista en tanto alude al concepto de que lo primero que ocurre es la investigación básica en los laboratorios y luego una secuencia de etapas que finalizan con la comercialización de la innovación lograda, comienza a ser cuestionado por otros modelos interpretativos. Al respecto, se pone en duda la idea de que el conocimiento debe partir de la ciencia básica para dar lugar a la noción de que es la demanda del mercado la que origina la creación del conocimiento.
} 


\section{CIENCIA Y TECNOLOGÍA EN ALC: ASPECTOS CARACTERÍSTICOS}

ALC comienza a desarrollar su complejo de CyT durante la posguerra, en el marco de un contexto socio-histórico en el que los países del mundo comenzaban a otorgar un lugar fundamental a estas actividades en el desarrollo económico, social y productivo. Sin embargo, el incipiente diseño del SCT latinoamericano fue tomando rasgos particulares en función de las características estructurales de las sociedades latinoamericanas. Estos rasgos que adquirió el SCT latinoamericano fue condicionante, pero a su vez también condicionado, del desempeño de los actores que componen el sistema.

A continuación se describen en forma somera aspectos que se consideran otorgan rasgos particulares al SCT de la región:

- Carácter imitativo de los diferentes organismos públicos de promoción de la CyT: en la región, los primeros organismos en la materia fueron los llamados Consejos de Ciencia y Tecnología (CCT) que fueron creados en la década del sesenta. No obstante, países como Argentina y Brasil ya contaban para esos años con sus propios organismos, como son el Conselho Nacional de Pesquisa (CNPq), creado en $1951^{3}$, y el Consejo Nacional de Investigaciones Científicas y Tecnológicas (CONICET), creado en 1958. Con relación a los CCT, Amadeo (1974) sostiene que en su creación se evidencia la influencia extranjera en la política de CyT en la región. La influencia extranjera a la que alude este autor refiere al importante papel que han tenido determinados organismos internacionales -como por ejemplo el caso de la UNESCOen la promoción de los modelos institucionales de los organismos de CyT en la región en la década del sesenta4 ${ }^{4}$. Por lo tanto, este carácter imitativo que han tenido los organismos públicos de promoción de la CyT incidió en las posibilidades de pensar desde los inicios en modelos institucionales más acordes a la realidad latinoamericana.

- Bajo desempeño del sistema de CyT en comparación con los países más desarrollados: a pesar de la relativa antigüedad del SCT en la región, el desempeño que el mismo ha tenido es muy bajo en comparación con los países más desarrollados. Este problema ya ha sido identificado en los años setenta y aún se repite en las evaluaciones comparativas de los países de la región. De acuerdo con Marí y Thomas (2000), los esfuerzos de los países latinoamericanos no lograron el despegue del sector científico y tecnológico -con algunas excepciones- y, en consecuencia, nunca llegó a ser atractivo para la demanda de tecnología del sector productivo. Las demandas del sector privado fueron casi siempre satisfechas por proveedores extranjeros de tecnologías a través de la venta de equipos o de plantas "llave en mano".

- Baja participación del sector privado en el financiamiento de la I+D en comparación con los países más desarrollados: desde sus orígenes y hasta la actualidad, el SCT latinoamericano se sostiene primordialmente gracias al aporte del Estado. Esto pone en evidencia otro rasgo del sistema, que es la baja participación del sector privado en

\footnotetext{
${ }^{3}$ A partir de 1971 pasa a denominarse Conselho Nacional de Desenvolvimento Científico e Tecnológico.

${ }^{4}$ Como se indicó ut supra, el modelo predominante en la producción de conocimiento era de tipo ofertista, en cuya base se encontraba la ciencia básica.
} 
el financiamiento de la I+D. Este comportamiento pasivo del empresariado se consolidó aún más con los procesos de apertura y trasnacionalización de la economía en los años noventa, ya que las empresas comienzan a importar paquetes tecnológicos completos y los equipos de investigación industrial comienzan a perder espacios (Marí y Thomas, ibíd.). Como sostiene Vaccarezza (1998), no es que no existieran vínculos entre ciencia y producción sino que el problema reside en que no constituye un sistema autosostenido de relaciones dinámicas que definan un rumbo claro a la CyT y sus vínculos con las sociedades y economías en las que se desenvuelven.

- Escasa articulación entre los organismos de CyT y la sociedad: este problema ya fue identificado en los años sesenta y setenta por muchos investigadores. Entre ellos se destacan Sábato y Botana (1968), quienes propusieron el reconocido modelo del "triángulo de las interacciones" 5 como orientador de las estrategias de desarrollo en la región. Para estos autores, las interacciones planteadas en el triángulo eran inexistentes en ALC, por lo que consideraban necesario no solo la creación de infraestructura científico-tecnológica sino la movilización de inteligencia y voluntad, para lo cual creían en la tarea decisiva de la universidad, que debía relacionarse necesariamente con el sector productivo. En esta línea, Herrera (1995 [1973]) sostiene que la falta de conexión entre la producción científica y las necesidades sociales de la región es una problemática constante en el SCT. Al respecto, este autor destacaba que el atraso científico y tecnológico regional, en comparación con los países más desarrollados, se debe a aspectos culturales e institucionales relacionados con el sistema de producción.

- Rasgos particulares de las comunidades científicas locales: una de las principales críticas sobre este punto lo realizó Varsavsky (1969), quien utilizó el término "cientificismo" para caracterizar la actividad científica local en el sentido de que muchos científicos se han convertido en productores de papers, convencidos en la necesidad de cuantificación como modo de otorgar verosimilitud a sus producciones pero sin que ello conduzca a producir nuevas ideas. El cientificismo explica la indiferencia del científico antes las problemáticas sociales que lo rodean puesto que los profesionales son formados en la ciencia como elemento de estudio apartado de la realidad. Para Varsavsky, esto también constituye una estrategia que contribuye al posicionamiento jerárquico de los países según su importancia en el sistema mundial, ya que el Hemisferio Norte impone internacionalmente los objetos de estudio y que el resto de los países -los subdesarrollados- lo adoptarán como tales. Sin embargo, no sólo es posible observar a las comunidades científicas locales por las lógicas de su campo (en el sentido bourdeano del término), sino también por el tránsito que estas comunidades han tenido durante los gobiernos militares en la región. Como indica Lovisolo (1996), las comunidades científicas en Argentina se desarrollaron tempranamente, pero sufrieron un retraimiento producto de la violencia sufrida por parte de los militares en el poder. Esto no ocurrió en Brasil, donde la interacción entre intelectuales, estado y élites fue mucho menos conflictiva.

\footnotetext{
${ }^{5} \mathrm{El}$ "Triángulo de las Interacciones" es un modelo analítico utilizado por los autores para representar las interacciones virtuosas entre el gobierno, los organismos de ciencia y tecnología y la estructura productiva. Relaciones dinámicas entre estos actores conducirán, según los autores, al desencademiento de procesos de innovación.
} 
- Pérdida de capacidades científico-tecnológicas por la "fuga de cebreros": la sucesión de gobiernos militares y las propias crisis económicas recurrentes en la región han provocado que países con población altamente calificada -como Argentina y Brasil- sufrieran durante mucho tiempo procesos de diáspora de sus profesionales (fenómeno conocido como "fuga de cerebros"), lo que afectó indudablemente al desarrollo de las capacidades tecnológicas de estos países (Didou Aupetit, 2009). Como sugiere Balán (2009), aún se desconoce la magnitud del impacto que provocan los mercados estudiantil y laboral de los centros de investigación de países como Estados Unidos, Gran Bretaña y otras potencias científica-tecnológicas en la formación y retención de académicos e investigadores de ALC.

\section{CARACTERIZACIÓN DE LOS SISTEMAS DE CYT EN ARGENTINA Y BRASIL}

A continuación, se describe someramente una serie de aspectos que refieren a la CyT en Argentina y Brasil. Los indicadores utilizados fueron consultados de la RICyT, permitiendo analizar no sólo la magnitud de las actividades de CyT sino también realizar una evaluación en términos comparativos con los países más avanzados que definen la frontera del conocimiento científico y el desarrollo tecnológico.

\subsection{Gasto en CyT en relación con el PBI (en \%)}

En Brasil, el gasto en CyT supera el 1\% y su crecimiento ha sido del 21\% entre los años 2000 y 2013. En el caso de Argentina, este valor se ubica en los últimos años de la serie en torno al o,60\%, presentando un crecimiento significativo del $42 \%$ en la última década. El promedio del gasto para ALC se encuentra por encima del $0,60 \%$, mientras que el valor promedio en los países más avanzados es del $2 \%$.

\subsection{Gasto en $I+D$ por sector de financiamiento}

En lo que refiere al sector de financiamiento del gasto en I+D, observamos que, en el caso de Argentina, el gobierno financia más del 60\% de las actividades en CyT mientras que el sector empresario -tanto público como privado- participa con menos de un tercio. Entre 2004-2008, el gobierno acentuó su participación en el gasto mientras que el sector privado la redujo. En Brasil la relación es un poco más equilibrada ya que el gobierno financia poco más del 50\% del gasto en I+D y el sector empresario participa con más del 40\%. Al igual que Argentina, la participación del gobierno en el gasto en $\mathrm{I}+\mathrm{D}$ ha crecido en los últimos años mientras que el sector empresario se ha retraído. En los países más avanzados, esta relación es inversa. 
El sector privado financia más del $60 \%$ del gasto en $\mathrm{I}+\mathrm{D}$ mientras que el gobierno lo hace en valores por debajo del $30 \%$.

\subsection{Investigadores por cada mil habitantes de la PEA (EJC)}

La cantidad de investigadores $\mathrm{EJC}^{6}$ por cada mil habitantes de la PEA de Argentina presenta mejores valores en comparación con Brasil e incluso muy superiores al promedio de ALC. En 2013, en Argentina, este valor era de 2,95 investigadores EJC cada mil habitantes de la PEA, mientras que en Brasil, para el año 2010 (último valor disponible), este valor era de 1,48 . Ambos países presentan un crecimiento sostenido en el número de investigadores EJC en la última década, lo cual demuestra el esfuerzo sostenido por mejorar las capacidades científico-tecnológicas. En los países más avanzados, los investigadores EJC ascienden a más de 7 cada mil habitantes de la PEA.

\subsection{Investigadores por sector de empleo (EJC)}

En 2013, el principal ámbito de inserción de los investigadores EJC en Argentina eran el sector gubernamental $(46,16 \%)$ y la educación superior $(45,72 \%)$, mientras que en el sector empresario el porcentaje es muy bajo (menos del 10\%). En Brasil, para el año 2010 (último dato disponible), los principales ámbitos de inserción son la educación superior (67,79\%) y el sector empresario (25,94\%). En ambos países ha disminuido la capacidad de absorción de investigadores por parte del sector empresario, lo que explica el énfasis otorgado en sus políticas de CyT a la necesidad de incrementar la inserción de los investigadores en las empresas. En los países desarrollados, el principal ámbito de inserción laboral de los investigadores es el sector empresario, tanto público como privado.

\section{LAS POLÍTICAS PÚBLICAS DE CYT EN CLAVE COMPARADA: BREVES REFLEXIONES}

$\mathrm{Al}$ momento de evaluar las políticas de CyT, uno de los mecanismos más utilizados es el de clasificar el momento en que se realiza el proceso de evaluación en términos de ex ante, intermedia y ex post (Osuna et al., 2003). La evaluación ex post, que es el tipo que evaluación que nos interesa en este trabajo, (...) se realiza una vez finalizada la política, el plan, el pro-

\footnotetext{
${ }^{6}$ Según la RICYT (2015: 21), la Equivalencia a Jornada Completa (EJC) es “(...) una medida que facilita la comparación internacional ya que se trata de la suma de las dedicaciones parciales a la investigación que llevan a cabo los investigadores durante el año, divididas por el número de horas de una dedicación completa a la I+D durante un año. Es decir, refiere con mayor precisión al tiempo dedicado a la investigación y resulta de particular importancia en sistemas de ciencia y tecnología en los que el sector universitario tiene una presencia preponderante, como es el caso de los países de América Latina, dado que los investigadores dedican una parte de su tiempo a la I+D y otra a la docencia o la transferencia".
} 
grama o el proyecto de $I+D+i$ en cuestión, con el objetivo de determinar su éxito, así como los principales resultados e impactos que ha producido" (Osuna, ibíd., p. 24). Como precisan Albornoz y Alfaraz (2008), la evaluación de resultados y la evaluación de impacto son dos cosas distintas. En primer lugar, la evaluación de resultados remite al cumplimiento de los objetivos y metas propuestas, mientras que la evaluación de impacto remite en que los eventuales resultados dan lugar a modificaciones en el entorno del objeto.

En lo que refiere a las evaluaciones de resultados de los programas de becas de posgrado, D’Onofrio y Gelfman (2010) indican que la métrica frecuente son la producción de artículos científicos en revistas internacionales con referato y la participación en patentes de invención. Se trata de captar el nivel de producción de ambos "productos" para un periodo determinado, atribuyendo el comportamiento de estas variables a los cambios cuantitativos y cualitativos en la población de profesionales con formación de posgrado.

Esta forma de evaluar resultados tiene su origen en el modelo económico de "insumosproductos" utilizado en la cienciometría, cuya lógica responde a que ciertos insumos (recursos humanos, capital, equipos, etc.) alimentan una "caja negra" de donde emergen ciertos productos como resultado de los insumos (Velho, 1994).

\section{POLÍticas PÚblicas ORIENTAdAS A PROMOVER LA FORMACIÓN DOCTORAL}

\subsection{El caso de Argentina: recuperación y fortalecimiento de la formación docto- ral en la última década}

En Argentina, el principal mecanismo público orientado a la formación de nivel doctoral son los programas de becas destinados a los graduados universitarios jóvenes y a los docentes de las Universidades Nacionales (UU.NN.). Estos programas de beca son financiados principalmente por el CONICET y la Agencia Nacional de Promoción Científica y Tecnológica (ANPCyT). En menor medida, las UU.NN. y otras agencias nacionales y provinciales también financian programas de becas de nivel doctoral.

El sistema de posgrado nacional está regulado por la Ley de Educación Superior $\mathrm{N}^{\circ} \mathbf{2 4 . 5 2 1}$ del año 1995 y su modificatoria, la Ley $\mathrm{N}^{\circ} 25.754$ del año 2003. En líneas generales, estas normativas establecen que las UU.NN. tienen la potestad para crear carreras universitarias de grado y posgrado, pudiendo este último tipo de formación desarrollarse exclusivamente en instituciones universitarias y en centros de investigación e instituciones de formación profesional superior de reconocido nivel y jerarquía, que haya suscripto convenios con las universidades. Las carreras de posgrado -especialización, maestría o doctorado- deberán ser acreditadas por la Comisión Nacional de Evaluación y Acreditación Universitaria (CONEAU). 
La formación de recursos humanos de alta calificación ha sido una de las principales líneas de acción del Plan Estratégico Nacional de Ciencia, Tecnología e Innovación "Bicentenario" (2006-201O), en el marco de decisiones políticas que otorgaron nuevamente a la CyT un rol estratégico en el desarrollo nacional. En ese plan estratégico, el objetivo con respecto a los recursos humanos consistía en incrementar el número de investigadores y becarios EJC sobre una base de 2.000 por año y lograr un mayor equilibrio en la redistribución regional de los recursos en CyT.

Posteriormente, en el Plan Nacional de Ciencia, Tecnología e Innovación 2012-2015 se afianza esta línea de acción, aunque la prioridad está en corregir la falta de orientación en la asignación de recursos humanos por áreas estratégicas, la necesidad de una mayor radicación de los investigadores en las empresas (según estimaciones, el $89 \%$ se radica en el sector público mientras que el $11 \%$ restante lo hace en las empresas) y resolver la alta concentración territorial de los recursos humanos dedicados a CyT en general y a la I+D en particular (el $72 \%$ se concentra en las provincias de Buenos Aires, Córdoba, Santa Fe y la Ciudad de Buenos Aires).

La meta propuesta con respecto a los recursos humanos planteada en el Plan Estratégico Nacional de Ciencia, Tecnología e Innovación "Bicentenario" (2006-2010) ha sido cumplida satisfactoriamente. El CONICET, que es la principal institución formadora de investigadores, a partir del año 2002 incrementó significativamente el número de becas otorgadas7. Para el periodo 2000-2010 el número de becas se incrementó en un 310\%, pasando de un total de 1.982 en el año 2000 al número de 8.122 en el año 2010. En los primeros años de la serie hubo un estancamiento en la incorporación de becarios (incluso de valor negativo entre 2001 y 2002), comportamiento que se revierte a partir del 2004 y que se consolida en los años sucesivos. En cuanto a la distribución de las becas, se verifica que las de tipo doctoral crecieron significativamente desde el 2004 y hasta el 2008 inclusive, mientras que las becas de tipo posdoctoral presentan una tasa de crecimiento irregular.

Esta política de ampliación de las becas claramente favoreció el incremento en el número de doctores. Según datos disponibles por la RICYT, entre 2004 y 2013 la población de doctores creció en un 356,89\%. El mayor incremento porcentual se produjo en el área de las Ciencias Agrícolas, donde se evidenció un crecimiento de casi 1000\%. En las Ciencias Humanas el crecimiento fue del 522,86\%; en las Ciencias Exactas y Naturales el aumento fue del 471,33\%; en el campo de la Ingeniería y Tecnología del 285\%; en las Ciencias Sociales del 275\%, y; por último, en las Ciencias Médicas del 183,82\%.

Los doctores de las Ciencias Exactas y Naturales representaban en 2013 el 41,02\% del total poblacional, mientras que los doctores de las Ciencias Sociales significaban el 28,20\%. En menor medida, las Ciencias Humanas representaban el 10,44\%, seguido por las Ciencias Médicas con el 9,24\%, la Ingeniería y Tecnología con el 7,37\% y, por último,

\footnotetext{
${ }^{7}$ Además del crecimiento sostenido en las becas, también se observa una mejora sustancial en el presupuesto del organismo y en lo que hace a los estipendios de las becas y de los salarios del personal científico y técnico. Asimismo, en estos años también se incrementó el número de investigadores a la Carrera del Investigador Científico y Tecnológico (CIC).
} 
las Ciencias Agrícolas representaban el 3,68\% del total. En la evolución de este periodo, las Ciencias Exactas y Naturales y las Ciencias Sociales tradicionalmente concentran el mayor número de doctores.

Ahora bien, sobre el total de titulados de posgrado, los doctores representaban en 2004 el 31,91\% del total; este porcentaje se elevó al 40,37\% en 2013. A pesar de este incremento, la población de posgraduados representaba para ese año tan sólo el 5\% del total de titulados universitarios.

\subsection{El caso de Brasil: política pública sostenida por más de sesenta años}

De acuerdo con Luchilo (op. cit.), el caso brasileño contrasta con el resto de los países de ALC no sólo por la cantidad de posgraduados sino por la continuidad de la política y su integración con las políticas de educación y de CyT. Para Balbachevsky (2005), el éxito de los programas de posgrado se inicia en los años setenta con un cambio en la política brasilera de CyT sobre la base de un consenso tácito entre dos elites: los líderes científicos y las fuerzas armadas. Según la autora, la formación de posgrado era esencial para el cumplimiento de los proyectos de ambos grupos. El gobierno lanzó un proyecto de formación de investigadores en el exterior que, una vez formados, retornaron al país y otorgaron una dinámica particular al sistema de posgrado brasilero (Balbachevsky, ibíd.).

Los principales organismos públicos responsables de promover la formación de recursos humanos son el CNPq y la Coordenação de Aperfeiçoamento de Pessoal de Nível Superior (CAPES). Al igual que la CNPq, la CAPES se crea en el año 1951. A mediados de la década de los setenta, la expansión de los programas de beca se produce conjuntamente con el desarrollo del Sistema de Evaluación de la Posgraduación por parte de la CAPES. A partir de ese periodo este organismo se consolida como agencia de promoción de la formación de posgrado, mientras que la CNPq se focaliza más en operar como unidad de financiamiento de la CyT del país.

Interesa observar que la política de formación de recursos humanos de alta calificación ha sido uno de los ejes del Plan Plurianual 2008-2011 del Ministerio de Ciencia y Tecnología brasileño. Al respecto, se han definido como líneas de acción el incremento de becas de formación, investigación y extensión con foco en las ingenierías y en las áreas consideradas prioritarias y la promoción de la inserción de ingenieros y doctores en las empresas, entre otros aspectos.

De acuerdo a los datos presentados por la RICYT, entre los años 2004-2013 la población total de doctores creció en un $88,89 \%$. Los mayores incrementos porcentuales se produjeron en las Ciencias Agrícolas, con un incremento del 110,83\%, seguido por las Ciencias Médicas con un incremento del 99,58\%. Siguen las Ciencias Humanas con un incremento del 82,65\%; las Ciencias Sociales con el 74,96\%; las Ciencias Naturales y Exactas con el 61,06\%, y; por último, la Ingeniería y la Tecnología con el 55,86\%. 
En 2013, el 33,07\% de los doctores correspondía al área de las Humanidades, seguido por las Ciencias Médicas con el 19,08\%, las Ciencias Agrícolas con el 13,43\%, la Ingeniería y Tecnología con el 10,25\%, las Ciencias Exactas y Naturales con el 9,52\% y, por último, las Ciencias Sociales con el 8,64\%. El peso de las distintas áreas sobre el total se ha mantenido en la última década, aunque las Ciencias Agrícolas y las Ciencias Médicas han ampliado levemente su participación sobre el total.

Ahora bien, si tomamos en cuenta el peso de los doctores sobre la población de posgraduados, observamos que en 2004 los doctores representaban el 54,86\% mientras que los magíster el 45,14\% restante. Esta relación se invierte considerablemente en 2013 a raíz del fuerte impulso por parte de la CAPES de las carreras de maestría de perfil profesionalista (Fliguer y Accinelli, 2011). En ese año, los graduados con maestría representaban el $76,63 \%$ del total mientras que los doctores el 23,36\% restante. Este nuevo panorama modificó el peso de los posgraduados sobre el total de titulados universitarios. En 2004 los titulados con maestría y doctorado representaban apenas el $2 \%$ del total, mientras que en 2013 este porcentaje se incrementa al $7 \%$.

En 2004, Brasil se encontraba entre los diez países con mayor número de doctores, aunque su posición descendía si se comparaba estos valores por cada 100 mil habitantes. A pesar del incremento sostenido de la población de doctores, la inserción laboral de estos profesionales demuestra que el sector público (principalmente la educación) absorbe a la mayor cantidad de doctores, mientras que en la industria sólo trabaja el 1,24\% (CGEE, 2008).

\subsection{Investigadores por nivel de formación en Argentina y Brasil: diferencias y similitudes}

Como se demostró para el caso de Argentina y Brasil, el número de doctores en ambos países creció en la última década. Si bien es un dato significativo en sí mismo, interesa también conocer si las personas dedicadas a la investigación han incrementado su formación. El dato es una medida indirecta del nivel de producción en CyT, al mismo tiempo que da cuenta del grado de profesionalización de las comunidades científicas de ambos países.

En Argentina, entre 2004 y 2013, los investigadores con doctorado se incrementaron en un $17,95 \%$, mientras que la población de investigadores con maestría se mantuvo constante. En 2013, más del 50\% de los investigadores en Argentina posee sólo título de grado. Es decir, si bien el país ha demostrado un crecimiento notable de la población de doctores en los últimos años, este incremento ha sido poco significativo si tomamos en consideración únicamente a las personas dedicadas a la investigación. Los datos disponibles para Brasil llegan hasta 2010. En el periodo 2004-2010, los investigadores con formación doctoral se han incrementado en un $17,94 \%$, mientras que los investigadores con formación en maestría aumentaron en un 9,19\%. En 2010, la población con formación de grado era aproximadamente el 20\%, valor con pendiente negativa en el periodo considerado. 
De este modo, y a partir de la lectura de los datos disponibles, la población de investigadores de Brasil presenta grados de profesionalización más altos que la Argentina.

\section{EVALUACIÓN DE RESULTADOS DE LAS POLÍTICAS PÚBLICAS ORIENTADAS A LA FORMACIÓN DE NIVEL DOCTORAL EN EL SISTEMA DE CIENCIA, TECNOLOGÍA E INNOVACIÓN}

Como se referenció ut supra, las publicaciones en revistas con referato y las solicitudes de patentes son dos indicadores de producto cuya dinámica se explican de acuerdo a la productividad de los recursos humanos dedicados a la CyT. De esta manera, el comportamiento de estos indicadores permite aproximarnos a comprender el impacto de las políticas públicas orientadas a la formación de nivel doctoral sobre el sistema de CyT argentino y brasilero.

Los indicadores de producto utilizados son:

\section{Publicaciones en $\mathrm{SCl}$}

Es un indicador que permite medir el factor de impacto ${ }^{8}$ de los textos citados en revistas indexadas que se encuentran en la base documental del Science Citation Index (SCI). De acuerdo con Sancho (2002), la producción científica de un país se calcula contando el número de publicaciones que dan cuenta de los resultados científicos alcanzados en ese país. Este valor variará de acuerdo al tamaño del país y el nivel de inversión en I+D. Este error se corrige calculando la productividad científica, que es la relación entre el total de publicaciones y el número de habitantes o investigadores.
Solicitudes de patente

Mide la creatividad tecnológica de un país o región a través de la solicitud de patentes de sus inventores. Este indicador no tiene necesariamente correlación con el otorgamiento de la patente. El indicador se desagrega en solicitud de "residentes" y "no residentes". La relación entre ambos grupos permite construir varias tasas, como por ejemplo la de dependencia tecnológica (número de patentes de inventores no residentes en el país, en relación con el número de patentes de residentes) y el coeficiente de invención (relación entre patentes solicitadas por residentes y la población total del país. Se expresa en patentes cada cien mil habitantes y descansa sobre el supuesto de que cuanto mayor sea el valor de este indicador, mayor es la capacidad de invención del país).

\subsection{Publicaciones en SCI}

En el periodo 2000-2013, el número de publicaciones en SCI para Argentina creció en un 95\%. A partir de 2005 los valores fueron incrementándose significativamente, aunque es recién en 2008 -que es el año de fortalecimiento del financiamiento de las actividades en CyTcuando las publicaciones registradas en SCI adquieren valores significativos. Este incremento se explica por los incrementos en el gasto en $\mathrm{I}+\mathrm{D}$, por la mayor incorporación de investigadores y becarios al sistema de CyT y por una mayor tasa de graduación de doctores.

En el caso de Brasil, el crecimiento de las publicaciones en SCI para el periodo 2000-2013 fue del 245,32\%. Como se observa en el gráfico 1, el salto cuantitativo se produce en 2006, que

\footnotetext{
${ }^{8}$ El factor de impacto se calcula según el número de artículos publicados en un año, en relación con las citas que han recibido esos mismos artículos en los dos años siguientes a su publicación.
} 
es el año en el cual se incrementa el gasto I+D del país (en este año Brasil retoma el sendero de invertir el 1\% de su PBI en actividades de I+D) y la tasa de posgraduados.

La diferencia en valores absolutos con Argentina se explica por el tamaño que tiene la comunidad científica en Brasil y las proporciones del gasto en I+D entre ambos países. En términos comparativos, el crecimiento de las publicaciones en SCI para el mismo periodo en ambos países demuestra un mayor nivel de producción de los investigadores brasileños en comparación con los argentinos.

Gráfico 1. Publicaciones en SCI de Argentina, Brasil y ALC. Periodo 2000-2013.

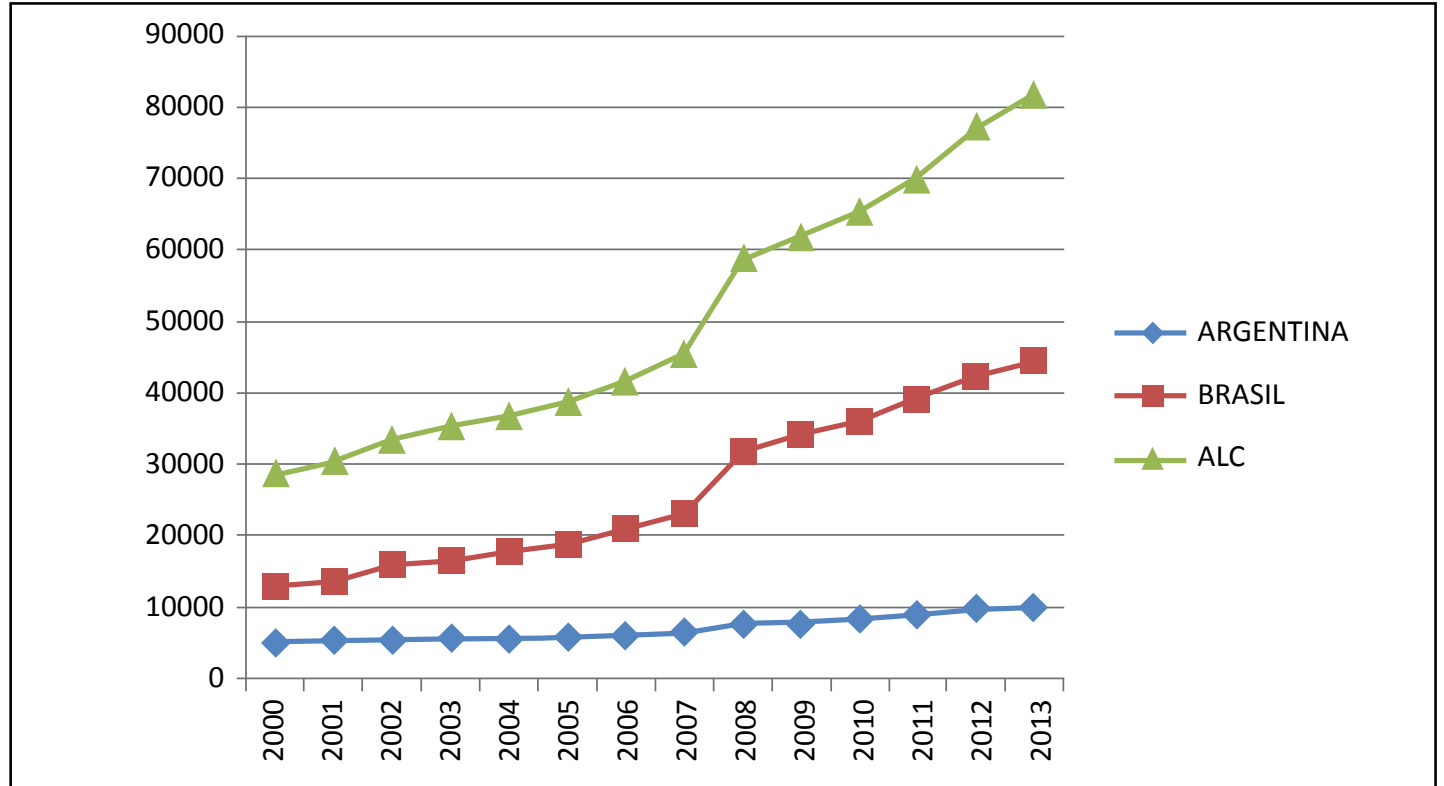

Fuente: elaboración propia en base a la RICYT (2016)

Sin embargo, esta diferencia numérica necesariamente no alude a una mayor productividad. Para ello es necesario observar la relación de las publicaciones en SCI por cada 100 investigadores EJC. Como se observa en el gráfico 2, Argentina y Brasil tuvieron niveles de productividad similares entre los años 2000 y 2007, aunque a partir de 2008 Brasil experimenta un crecimiento significativo en este indicador mientras que Argentina, a pesar de los esfuerzos de inversión en $\mathrm{I}+\mathrm{D}$ y en formación de recursos humanos, no ha modificado significativamente los valores. Si bien para Brasil solo se disponen de datos hasta el año 2010, la lectura de los datos nos permite demostrar que este país incrementó no sólo la producción sino también la productividad de su comunidad científica, mientras que Argentina experimentó un crecimiento en la producción pero no en la productividad. 


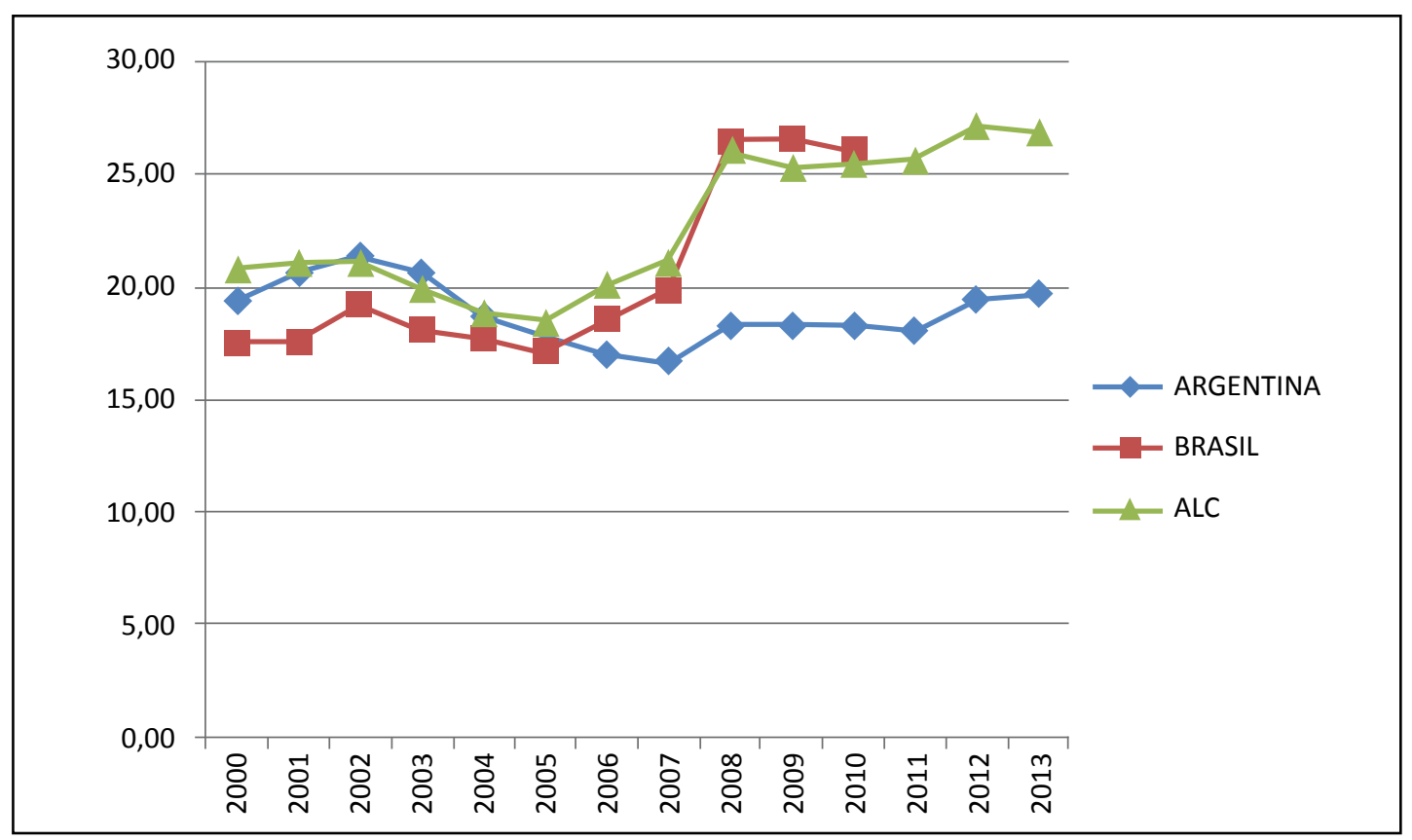

Fuente: elaboración propia en base a la RICYT (2016)

\subsection{Solicitudes de patentes}

En Argentina, la solicitud de patentes de los "residentes" presenta tendencia negativa entre los años 2004-2013. Si tomamos los valores de 2004 y 2013, la disminución fue del 13\%. Por su parte, la solicitud de patentes de los "no residentes" creció un 8,20\% en el periodo considerado. En Brasil, la solicitud de patentes de los "residentes" creció un 3,54\% entre 2004 y 2013, mientras que la solicitud de patentes de los "no residentes" experimentó un crecimiento del 104,91\% para este mismo periodo.

Tabla 1. Solicitud de patentes de residentes y no residentes de Argentina, Brasil y ALC. Periodo 2004-2013

\begin{tabular}{|c|c|c|c|c|c|c|c|c|c|c|c|}
\hline & Solicitudes de Patentes & 2004 & 2005 & 2006 & 2007 & 2008 & 2009 & 2010 & 2011 & 2012 & 2013 \\
\hline \multirow[t]{3}{*}{ Argentina } & de residentes & 786 & 1054 & 1020 & 937 & 801 & 640 & 552 & 688 & 697 & 643 \\
\hline & de no residentes & 3816 & 4215 & 4597 & 4806 & 4781 & 4336 & 4165 & 4133 & 4119 & 4129 \\
\hline & Total & 4602 & 5269 & 5617 & 5743 & 5582 & 4976 & 4717 & 4821 & 4816 & 4772 \\
\hline \multirow[t]{3}{*}{ Brasil } & de res & 7701 & 7346 & 7194 & 7326 & 7711 & 7709 & 244 & 7797 & 7808 & 7974 \\
\hline & de no & 12725 & 14470 & 15937 & 17496 & 18905 & 18144 & 20825 & 24055 & 25724 & 26075 \\
\hline & \begin{tabular}{|l|} 
Total \\
\end{tabular} & 20426 & 21816 & 23131 & 24822 & 26616 & 25853 & 28069 & 31852 & 33532 & 34049 \\
\hline \multirow[t]{3}{*}{ ALC } & de res & 10297 & 10138 & 9988 & 9981 & 10363 & 10132 & 9670 & 10569 & 10811 & 10915 \\
\hline & de no residentes & 39595 & 45019 & 49724 & 52273 & 54028 & 46404 & 48292 & 52803 & 55460 & 55606 \\
\hline & Total & 49892 & 55157 & 59712 & 62254 & 64390 & 56536 & 57961 & 63373 & 66271 & 66521 \\
\hline
\end{tabular}

Fuente: elaboración propia en base a la RICYT (2016)

Los valores observados de ambos países respecto de la solicitud de patentes de "residentes" ponen en evidencia la diferencia en el grado de creatividad y desarrollo tecnológico. 
En Argentina, este valor es negativo en términos globales -aunque con años de incremento en las solicitudes - mientras que en Brasil es positivo casi en toda la serie histórica considerada. De este modo, a pesar de que Argentina fortaleció su sistema de I+D e incrementó su dotación de doctores, los resultados tecnológicos obtenidos son prácticamente nulos. Por su parte, Brasil, si bien experimentó un incremento en las solicitudes, tampoco presenta incrementos sostenidos si tomamos en consideración la envergadura de su desarrollo científico en la última década.

Ahora bien, en ambos países la solicitud de patentes de "no residentes" se incrementó en el periodo considerado, superando ampliamente a la solicitud de los "residentes". Estos valores se traducen en la tasa de dependencia tecnológica y en el coeficiente de invención. Como se mencionó anteriormente, la tasa de dependencia tecnológica expresa la relación entre el número de patentes de inventores no residentes en el país, en relación con el número de patentes de residentes. En la tabla 3 se observa que la tasa de dependencia tecnológica en ambos países se ha incrementado en más de 1.5 puntos entre 2004 y 2013. Esto demuestra que, a pesar de que Argentina y Brasil han mejorado sus indicadores de producción bibliométrica, la creatividad tecnológica sigue siendo materia pendiente de desarrollo e inversión.

Tabla 1. Solicitud de patentes de residentes y no residentes de Argentina, Brasil y ALC. Periodo 2004-2013

\begin{tabular}{|l|c|c|c|c|c|c|c|c|c|c|}
\hline Años & $\mathbf{2 0 0 4}$ & $\mathbf{2 0 0 5}$ & $\mathbf{2 0 0 6}$ & $\mathbf{2 0 0 7}$ & $\mathbf{2 0 0 8}$ & $\mathbf{2 0 0 9}$ & $\mathbf{2 0 1 0}$ & $\mathbf{2 0 1 1}$ & $\mathbf{2 0 1 2}$ & $\mathbf{2 0 1 3}$ \\
\hline Argentina & 4,85 & 4,00 & 4,51 & 5,13 & 5,97 & 6,78 & 7,55 & 6,01 & 5,91 & 6,42 \\
\hline Brasil & 1,65 & 1,97 & 2,22 & 2,39 & 2,45 & 2,35 & 2,87 & 3,09 & 3,29 & 3,27 \\
\hline ALC & 3,85 & 4,44 & 4,98 & 5,24 & 5,21 & 4,58 & 4,99 & 5,00 & 5,13 & 5,09 \\
\hline
\end{tabular}

Fuente: elaboración propia en base a la RICYT (2016)

Por último, el coeficiente de invención (CI) nos indica la capacidad inventiva de un país, calculada a partir de la solicitud de patentes de los "residentes" con relación a la población total nacional. En Argentina, el CI disminuyó significativamente desde 2005 y hasta 2010, recuperándose a partir de 2011 aunque sin alcanzar los valores de 2005. En Brasil el CI presenta un comportamiento más estable en el periodo considerado, aunque el valor del año 2013 se encuentra por debajo del valor de 2004 .

Tabla 1. Solicitud de patentes de residentes y no residentes de Argentina, Brasil y ALC. Periodo 2004-2013

\begin{tabular}{|l|c|c|c|c|c|c|c|c|c|c|}
\hline Años & $\mathbf{2 0 0 4}$ & $\mathbf{2 0 0 5}$ & $\mathbf{2 0 0 6}$ & $\mathbf{2 0 0 7}$ & $\mathbf{2 0 0 8}$ & $\mathbf{2 0 0 9}$ & $\mathbf{2 0 1 0}$ & $\mathbf{2 0 1 1}$ & $\mathbf{2 0 1 2}$ & $\mathbf{2 0 1 3}$ \\
\hline Argentina & 2,10 & 2,78 & 2,66 & 2,42 & 2,04 & 1,61 & 1,38 & 1,70 & 1,70 & 1,55 \\
\hline Brasil & 4,21 & 3,97 & 3,84 & 3,87 & 4,03 & 3,98 & 3,71 & 3,95 & 3,92 & 3,97 \\
\hline ALC & 1,90 & 1,84 & 1,79 & 1,78 & 1,82 & 1,76 & 1,65 & 1,78 & 1,80 & 1,79 \\
\hline
\end{tabular}

Fuente: elaboración propia en base a la RICYT (2016) 


\section{CONSIDERACIONES FINALES}

En este trabajo se analizaron dos indicadores de producto en CyT -las publicaciones en SCI y las solicitudes de patentes- con la intención de describir comparativamente el tipo de impacto que han tenido las políticas públicas de formación doctoral implementadas en Argentina y Brasil en el SNI. Si bien entendemos que los reales impactos de las inversiones en CyT se evidencian en el largo plazo, el análisis realizado permite construir algunas hipótesis explicativas sobre el campo de la producción científico-tecnológica.

Como se observa en los datos analizados, las políticas públicas llevadas a cabo en ambos países han favorecido el incremento en el número de doctores, aspecto que favoreció el aumento de las publicaciones científicas de excelencia de sus comunidades científicas. Ahora bien, Brasil presenta mejores indicadores en comparación con Argentina (en producción y productividad) no sólo porque su comunidad científica es más numerosa, sino también porque presenta una mayor inversión en $\mathrm{I}+\mathrm{D}$ y porque históricamente este país ha mantenido una fuerte estrategia de formación de profesionales de alto nivel, situación que no se ha visto interrumpida como ocurrió en el caso argentino donde los gobiernos militares, e incluso los democráticos, han favorecido el desmantelamiento del sistema científico-tecnológico.

No obstante, el incremento en el número de doctores en ambos países no ha impactado hasta el momento en términos de resultados tecnológicos (solicitudes de patentes). Al respecto, es importante comprender que el SCT latinoamericano se ha caracterizado estructuralmente por la baja inversión del gasto privado, la falta de demanda de conocimiento científico y tecnológico por parte del sector empresarial y la escasa articulación entre las instituciones públicas de CyT y el sector productivo. Esto demuestra que el sector empresarial ofrece pocas condiciones para la inserción de profesionales altamente calificados, aspecto que dificulta el desencadenamiento de innovaciones al interior de las firmas.

Por ello, y para finalizar, enfatizamos en la necesidad de realizar una lectura situada de los indicadores en cienciometría, comprendiendo que las prácticas que los mismos representan se inscriben en el marco de un contexto socio-económico y político que les otorga sentido. De este modo, esperamos haber contribuido en esta línea de discusión, deseando se enriquezca el análisis aquí presentado incluyendo nuevos y más indicadores que permitan comprender mejor la relación entre las políticas públicas de formación doctoral y la producción científicotecnológica. 


\section{REFERENCIAS BIBLIOGRÁFICAS}

Albornoz, Mario (2011). El estado de la ciencia 2011. Principales indicadores de ciencia y tecnología iberoamericanos / Interamericanos. Buenos Aires: RICYT. Disponible en http:// docs.politicascti.net/documents/Comparativos/Albornoz_EEC_2011.pdf

Albornoz, Mario y Alfaraz, Claudio (2008). "Diseño de una metodología para la medición del impacto de los centros de excelencia”. Documento de trabajo $\mathrm{N}^{\circ} 37$. Centro Redes, Buenos Aires, Argentina. Disponible en: http://www.centroredes.org.ar/files/documentos/Doc.Nro37.pdf

Amadeo, Eduardo (1978). "Los consejos nacionales de ciencia y tecnología en América Latina”, en Comercio Exterior, vol. 28, núm. 12, pp. 1439-1447.

Balán, Jorge (2009). "Los mercados académicos en el Norte y la migración internacional altamente calificada: el contexto actual de la circulación de cerebros de América Latina”, en DIDOU AUPETIT, S. Y ETIENNE, G. (Comp.), Fuga de cerebros, movilidad académica, redes científicas. Perspectivas latinoamericanas (pp. 75-88). México: IESALC-CINVESTAV-IRD. http://unesdoc.unesco.org/images/oo18/o01864/186433s.pdf

Balbachevsky, Elizabeth (2005). “A pós-graduação no Brasil: novos desafios para uma política bem-sucedida”, en BROCK. C.; SCHWARTZMAN, S., Os desafios da educação no Brasil (pp. 275-304). Rio de Janeiro: Nova Fronteira.

CENTRO DE GESTÃO E ESTUDOS ESTRATÉGICOS (2008). Características do Emprego dos Doutores Brasileiros: características do emprego formal no ano de 2004 das pessoas que obtiveram título de doutorado no Brasil no período 1996-2003. Brasilia, DF: CGEE.

D’onofrio, María Guillermina y Gelfman, Julia (2010). “Aspectos metodológicos de la evaluación de resultados e impactos de programas de formación de científicos y tecnólogos en los países iberoamericanos: dimensiones de análisis y fuentes de información”, en LUCHILO, L. (Comp.), Formación de posgrado en América Latina: políticas de apoyo, resultados e impactos (217-254). Buenos Aires: Eudeba.

Didou Aupetit, Sylvie (2009). “¿Pérdida de cerebros y ganancia de saberes?: la movilidad internacional de recursos humanos altamente calificados en América latina y el Caribe”, en DIDOU AUPETIT, S. Y ETIENNE, G. (Comp.), Fuga de cerebros, movilidad académica, redes científicas. Perspectivas latinoamericanas (pp. 25-62). México: IESALC-CINVESTAV-IRD. Disponible en: http://unesdoc.unesco.org/images/o018/001864/186433s.pdf 
Fliguer, José y Accinelli, Adriana (2011). Perspectivas en el proceso de integración de los sistemas de posgrados de Argentina y Brasil en el marco del MERCOSUR Educativo. Buenos Aires: Fundación de Ciencias Empresariales y Sociales (FUCES).

García Guadilla, Carmen (1996). Situación y principales dinámicas de transformación de la Educación Superior en América Latina. Caracas: Ediciones CRESAL/UNESCO.

GLOBAL SCIENCE FORUM (2008). Encouraging Student Interest in Science and Technology Studies. París: OCDE.

Herrera, Amílcar (1995 [1973]). "Los determinantes sociales de la política científica en América Latina. Política científica explícita y política científica implícita”, en Revista REDES, Vol. 2, $N^{o}$ 5, pp. 117-131. UNQ, Buenos Aires.

Jaramillo Salazar, Hernán (2009). "La formación de posgrado en Colombia: maestrías y doctorados", en Revista Iberoamericana de Ciencia, Tecnología y Sociedad, 5(13), pp. 131-155. Disponible en http://www.revistacts.net/files/Volumen\%205\%20\%20n\%C3\%BAmero\%20 13/jaramillo.pdf

Lovisolo, Hugo (1996). "Comunidades científicas y universidades en la Argentina y el Brasil”, en Revista REDES, Vol. III, núm. 8, diciembre, pp.47-94. UNQ, Buenos Aires. Disponible en http://www.redalyc.org/pdf/907/90711321002.pdf

Luchilo, Lucas (2010). "Programas de apoyo a la formación de posgrado en América Latina: tendencias y problemas”, en LUCHILO, L. (Comp.), Formación de posgrado en América Latina: políticas de apoyo resultados e impactos (pp. 13-32). Buenos Aires: Eudeba.

Marí, Manuel y Thomas, Hernán, (2000). Ciencia y Tecnología en América Latina. Bernal: Universidad Virtual de Quilmes.

MINISTÉRIO DA CIÊNCIA E TECNOLOGIA (MCT) (2008). Plano Plurianual 20082011: orientações estratégicas do Ministério da Ciência e Tecnologia. Brasilia: MCT.

Mollis, Marcela (2010). "Las transformaciones de la Educación Superior en América Latina: Identidades en construcción”, en Educación Superior y Sociedad, Vol. 15, No 1, pp. 11-23. Disponible en http://ess.iesalc.unesco.org.ve/index.php/ess/article/view/366/305 
Mollis, Marcela y Jaim Etcheverry, Guillermo (2003). "Posgrados universitarios: ¿actividad académica o servicio al cliente? El caso de la Universidad de Buenos Aires”, en Estudios de Posgrado. Perspectivas y desafíos $\mathrm{N}^{\circ}$ 18, pp. 261-277.

Osuna, J. L., Grávalos, E., Y Palacios, C. (2003). Modelos de protocolos para la evaluación de actividades de I+D e innovación. Madrid: Fundación Española para la Ciencia y la Tecnología (FECYT).

Rama Vitale, Claudio (2007). Los postgrados en América Latina y el Caribe en la sociedad del conocimiento. México: UDUAL.

RICYT(2015). El estado dela ciencia. OEI. Disponible en: http://www.ricyt.org/publicaciones

Sábato Jorge y Botana Natalio (1968). "La ciencia y la tecnología en el desarrollo futuro de América Latina”, en Revista de la Integración, INTAL, Año 1, n. ${ }^{\circ}$ 3, pp. 15-36. Buenos Aires.

Sancho Lozano, Rosa (2002). "Indicadores de los sistemas de ciencia, tecnología e innovación”, en Economía Industrial, Núm. 343, pp. 97-109.

SECRETARÍA DE CIENCIA Y TECNOLOGÍA (SECYT) (2006). Plan Estratégico Nacional de Ciencia, Tecnología e Innovación “Bicentenario" (2006-2010). Buenos Aires: MECyT.

Vaccarezza, Leonardo Silvio (1998). "Ciencia, tecnología y sociedad: el estado de la cuestión en América Latina”, en Revista Iberoamericana de Educación, Núm. 8 - Ciencia, tecnología y sociedad ante la educación. Disponible en: http://rieoei.org/oeivirt/rie18ao1.htm

Varsavsky, Oscar (1969). Ciencia, Política y Cientificismo. Buenos Aires: CEAL.

Velho, Lea (1998). "Indicadores científicos: aspectos teóricos y metodológicos e impactos en la política científica”, en MARTÍNEZ, E.y ALBORNOZ, M.(eds), Indicadores de ciencia y tecnología: estado del arte y perspectivas (pp. 23-51) Caracas: Nueva Sociedad. 


\section{CURRICULUM VITAE}

\section{Lucas Ariel Oviedo}

Licenciado en Relaciones Laborales (Universidad Nacional del Nordeste). Maestrando en Ciencia, Tecnología y Sociedad (Universidad Nacional de Quilmes). Doctorando en Ciencias Sociales (Universidad Nacional de Entre Ríos). Docente Investigador Categoría V en el Programa de Incentivos de la Secretaría de Políticas Universitarias (SPU) del Ministerio de Educación y Deportes de la Nación. Docente de la carrera de Relaciones Laborales de la Facultad de Ciencias Económicas de la UNNE. Becario del Consejo Nacional de Investigaciones Científicas y Técnicas (CONICET), con actividades de investigación en el Grupo I+D “Trabajo, Sociedad y Subjetividad" de la Facultad de Ciencias Económicas de la UNNE.

luc.oviedo@gmail.com 